\title{
Neuroretinitis: Update on a visual emergency and role of technology in its diagnosis
}

\author{
Subashini Kaliaperumal ${ }^{1}$, Sunil Narayan ${ }^{2 *}$ \\ ${ }^{1}$ Department of Ophthalmology, Jawaharlal Institute of Postgraduate Medical Education and Research, Pondicherry, India \\ ${ }^{2}$ Department of Neurology, Jawaharlal Institute of Postgraduate Medical Education and Research, Pondicherry, India \\ Email: *SUNIL.NARAYAN@JIPMER.EDU.IN
}

Received 24 July 2013; revised 28 August 2013; accepted 15 September 2013

Copyright (c 2013 Subashini Kaliaperumal, Sunil Narayan. This is an open access article distributed under the Creative Commons Attribution License, which permits unrestricted use, distribution, and reproduction in any medium, provided the original work is properly cited.

\begin{abstract}
Neuroretinitis is one of the forms of optic neuritis characterized by swelling of optic nerve head and adjoining retinal nerve fiber layer resulting in a macular star configuration. The underlying pathophysiology involves increased permeability of disc vasculature, but the etiology is not fully defined. Neuroretinitis may occur due to an infectious process involving the disc, a postviral or autoimmune mechanism or sometimes idiopathic. Technological advances like ophthalmoscopy, slit-lamp examination, fluorescein angiography, magnetic resonance imaging and immunodiagnostic tests all come handily and are often imperative in making an accurate diagnosis. Conditions mimicking neuroretinitis include papilledema, anterior ischemic optic neuropathy, and infiltration of the optic disc by tumor and systemic hypertension. Neuroretinitis is usually a self-limited disorder with a good visual prognosis. Treatment of neuroretinitis is required only when there is an underlying infectious or inflammatory condition.
\end{abstract}

Keywords: Neuroretinitis; Macular Star;

Ophthalmoscopy; Slit-Lamp; Fluorescein Angiography; Magnetic Resonance Imaging

\section{INTRODUCTION}

Theodor Leber way back in 1916 described this condition as stellate maculopathy [1]. Gass in 1977 coined the term neuroretinitis [2]. Neuroretinitis is a particular form of optic neuropathy characterised by acute unilateral visual loss in the setting of optic disc swelling and hard exudates arranged in a star figure around the fovea [3]. It affects persons of all ages, although it occurs more often

\footnotetext{
"Corresponding author.
}

in the third and fourth decades of life, with no gender predilection [4,5]. It is mostly unilateral and may be precipitated by various, known and unknown factors.

Neuroretinitis is a rare clinical entity often confused with the more common papillitis or papilledema. The fundus in these and other forms of opticneuropathy have several common features and can be misdiagnosed by the ill-experienced clinician and sometimes even by ophthalmologists and neurologists. However, there are certain diagnostic features of neuroretinitis. It is a distinct clinical entity with a definite etiopathogenesis. Likewise its management and prognosis too differs from fundoscopically similar entities such as papilledema and papillitis, which are encountered more often in our clinical practice.

Though the term neuroretinitis emphasizes clinical involvement of both disc and retina, the pathogenic locus is within the optic nerve head and macula is not the primary disease locus.

\section{CLINICAL PICTURE}

The clinical picture of neuroretinitis is characteristic and distinct from other optic neuropathies. This condition is usually painless but some patients complain of eye pain that may worsen with eye movements as seen in optic neuritis. If the neuroretinitis is due to an infectious process, there may be associated fever, malaise or headache. Patients usually present with a history of painless diminution of vision of acute onset. It is usually unilateral but may be bilateral in $5 \%-30 \%$ of the cases [5]. Visual acuity at presentation can range from 20/20 to light perception and is mostly due to the macular edema. A relative afferent papillary defect is present and is especially prominent in recurrent cases.

Fundus examination may show a few overlying vitreous cells. Optic disc appears hyperemic and edematous 
with peripapillary and macular edema. Initially macula has an opaque appearance due to edema. After 2 - 3 weeks the edema resolves leaving behind hard exudates. Since the edema is mostly confined the outerplexiform layer, macular star pattern of hard exudates forms (Figure 1). Sometimes cotton wool spots and splinter haemorrrhages may be seen. One feature commonly seen in catscatch disease associated neuroretinitis is the presence of yellow white deep retinal spots in the fundus. These are believed to be areas of colonization by the organism [6].

\section{PATHOPHYSIOLOGY}

The pathogenesis of neuroretinitis is obscure. It is related to direct involvement by aninfectious process or inflammation leading to edema of the optic nerve and cellular and fluid exudation from the inflamed area of peripapillary retina. The target tissue of the inflammatory response is the optic disc vasculature. Inflammation of the disc capillaries causes leakage of fluid and proteins into sub retinal space and outer plexiform layer. Resolution of fluid component leaves behind lipid exudates as a macular star [4].

\section{ETIOLOGY: (TABLE 1)}

Neuroretinitis is thought to be an infectious or immunemediated process that may be precipitated by a number of different agents. The common infections that cause neuroretinitis are cat-scratch disease, and the spirochetoses especially syphilis [7], Lyme disease, and leptospirosis [4]. Cat-scratch disease accounts for two thirds of cases [8,9]. Additional causes include toxoplasmosis [7], mumps [10], salmonella [11], tuberculosis [12], and

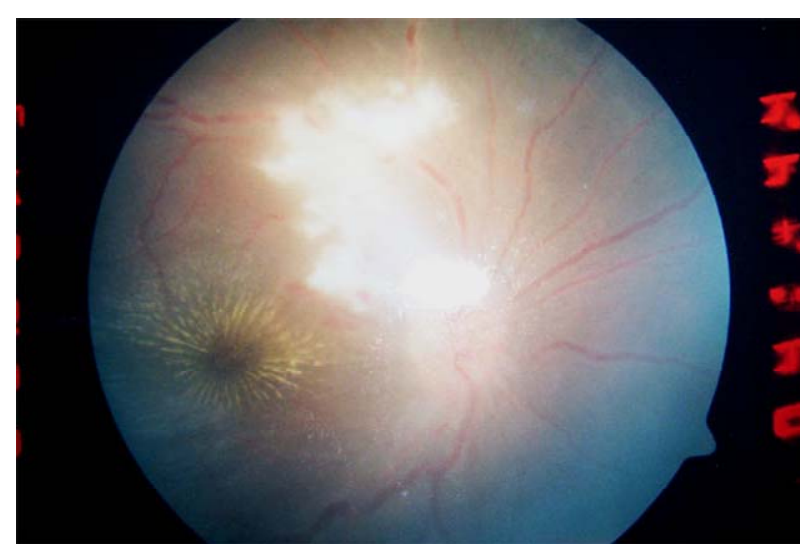

Figure 1. Neuroretinitis with vasculitis of the superotemporal vessel (reproduced from "Sunil K Narayan, Subashini Kaliaperumal, Renuka Srinivasan Neuroretinitis, a great mimicker. Annals of Indian Academy of Neurology, Year 2008, Volume 11, Issue 2 [pp. 109-113] DOI: 10.4103/0972-2327.41879 PMID: 19893649. by same authors with permission from publisher Wolters Kluwer Medknow 6.7.13”).
Table 1. Etiology of neuroretinitis.

Idiopathic (25\%)

Infectious:

1) Bartonella henselae (cat-scratch disease): most common cause ( $60 \%$ of the cases)

2) Toxoplasma gondii (toxoplasmosis)

3) Treponema pallidum (syphilis)

4) Borrelia burgdorferi (Lyme disease)

5) Leptospira spp. (leptospirosis)

6) Mycobacterium tuberculosis (tuberculosis)

7) Histoplasma capsulatum (histoplasmosis)

8) Rickettsia typhi (murine typhus)

9) Brucella spp. (brucellosis)

10) Viral etiologies: HIV, Varicella zoster virus, Herpes simplex virus, rarely hepatitis $\mathrm{B}$ or $\mathrm{C}$ virus

11) Nematodes: DUSN (Diffuse Unilateral Subacute Neuroretinitis) rarely

Parainfectious

Miscellaneous
1) Sarcoidosis
2) Inflammatory bowel disease
3) IRVAN (Idiopathic Retinal Vasculitis, aneurysms and neuroretinitis) syndrome

4) Poly arteritis nodosa

histoplasmosis. Rarely, a toxocaral granuloma within the optic nerve head produces a similar ophthalmoscopic picture [13]. Despite thorough evaluation, approximately one quarter of cases remain idiopathic.

Neuroretinitis is commonly associated with an antecedent viral syndrome, suggesting a possible viral etiology for upto $50 \%$ of the cases; however viruses are rarely cultured from the CSF of such patients, and serological evidence of a concomitant viral infection is usually lacking. Proposed causative viral agents include herpes simplex, hepatitis B, mumps, and the herpes viruses associated with the acute retinal necrosis syndrome. HIV with opportunistic infections especially syphilis and Hepatitis viruses have been implicated in neuroretinitis [14].

\section{RELATIONSHIP WITH MUTIPLE SCLEROSIS}

Multiple sclerosis is one condition that is not associated with neuroretinitis [4]. It is a well known fact that patients who develop typical optic neuritis are prone to develop multiple sclerosis but there is no similar increased 
tendency for patients who experience an attack of neuroretinitis [15]. Thus, when a diagnosis of an attack of acute optic neuropathy as an episode of neuroretinitis rather than anterior optic neuritis is made, it substantially alters the neurologic prognosis in the patient being evaluated. Nevertheless, there have been anecdotal reports of patients with multiple sclerosis who developed neuroretinitis [16].

\section{CONDITIONS MIMICKING NEURORETINITIS: (TABLE 2)}

Certain noninfectious and noninflammatory conditions mimick neuroretinitis as they are characterised by optic disc swelling that may on occasion be associated with the development of a macular star figure. These mimicking conditions include papilledema, anterior ischemic optic neuropathy, and inflitration of the optic disc by tumor [12]. Systemic hypertension may also produce both optic disc swelling and a macular star figure (Figure 2). The disk edema and retinopathy resolves after the hypertension is controlled [17]. Optic disc swelling in patients with systemic vascular disease like diabetes and hypertension can be differentiated form neuroretinitis by the absence of abrupt visual loss, background retinopathy and a medical history of such conditions. Spontaneous resolution of the disc edema and recovery of visual acuity serve as distinguishing features of neuroretinitis from papilledema and ischemic optic neuropathy.

\section{INVESTIGATIONS: (TABLE 3)}

Investigation into the etiology of neuroretinitis should begin with a careful history including questioning regarding sexually transmitted diseases, cat-scratches, skin rashes, tick bites, lymphadenopathy, fever, and flu-like illnesses. Complete physical and ocular examinations are essential. Screening with serological testing for treatable diseases such as cat-scratch disease, syphilis, and Lyme disease, analysis of CSF, neuroimaging may be desirable in the appropriate setting. In the absence of a proven etiology a diagnosis of Leber's idiopathic stellate neuroretinitis may be entertained.

Table 2. Differential diagnosis for disk edema with macular star [4].
1) Hypertensive retinopathy
2) Papilledema
3) Anterior ischemic optic neuropathy
4) Diabetic papillopathy
5) Branch retinal vein occlusion/papillophlebitis
6) Optic disk tumor: melanocytoma, juxtapapillary angioma

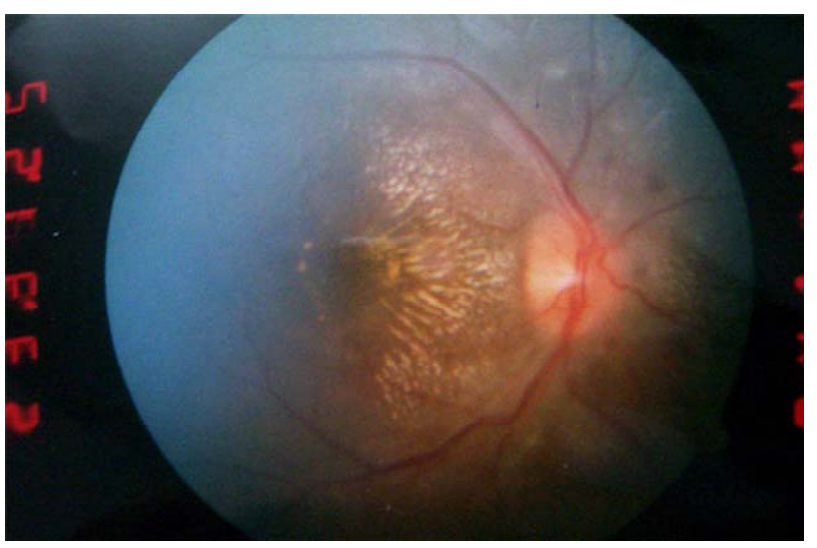

Figure 2. Grade IV hypertensive retinopathy mimicking as Neuroretinitis (reproduced from "Sunil K. Narayan, Subashini Kaliaperumal, Renuka Srinivasan Neuroretinitis, a great mimicker. Annals of Indian Academy of Neurology, Year 2008, Volume 11, Issue 2 [pp. 109-113] DOI: 10.4103/0972-2327.41879 PMID: 19893649. by same authors with permission from publisher Wolters Kluwer Medknow 6.7.13”).

Table 3. Suggested investigative workup in neuroretinitis.

\begin{tabular}{l}
\hline Ocular \\
\hline $\begin{array}{l}\text { Color vision, contrast sensitivity, central fields, fluorescein } \\
\text { angiography, VEP }\end{array}$ \\
\hline Systemic \\
\hline Blood culture-cat scratch disease \\
VDRL and FTA-ABS-Syphilis \\
Viral serology \\
Mantoux, chest X ray \\
ESR \\
Lumbar Puncture—opening pressure, cells, proteins, glucose, \\
CSF culture for bacteria especially leptospirosis and fungi \\
Immunofluorescent antibody test-cat scratch disease \\
ELISA-toxoplasmosis, toxocariasis \\
Polymerase chain reaction—cat scratch disease \\
Neuroimaging
\end{tabular}

\section{HOW DOES TECHNOLOGY HELP?}

\subsection{Ophthalmoscopy}

Direct ophthalmoscopy is the primary method of evaluation since this reveals striking changes in the optic fundi. Indirect ophthalmoscopy may help more detailed stereoscopic evaluation of the optic disc and retina.

\subsection{Visual Field Testing}

The visual field can be plotted using the Tangent screen or the Automated Humphrey perimetry. Typically the visual field defect noted will be a central or centrocaecal scotoma. Visual field defects are mainly due to maculopathy than optic neuropathy [18]. The degree of colour deficit is usually worse than the degree of visual loss would suggest. 


\subsection{Slit Lamp Biomicroscopy}

The slit lamp is an invaluable tool in the examination of the anterior segment and posterior segment of the eye. The two main components of the modern slit lamp microscope are the illumination system and the observation system. The illumination systems of today are capable of producing a homogenous, aberration-free beam of white light. Most slit lamps use halogen bulbs to yield shorter wavelengths of light which allow better visualization of smaller structures. The observation system allows magnification between $5 \times$ to $40 \times$. A slit lamp's resolution depends on the wavelength used, the refractive index of the eye and objective, the working distance and the diameter of the objective lens. To visualize the optic disc and the retina, as in neuroretinitis, special high convex lenses such +90 Dioptre lens are used with the slit lamp which allow stereoscopic view and give fine detail.

\subsection{Fluorescein Angiography}

Fluorescein angiography in patients with acute neuroretinitis demonstrates diffuse disc swelling and leakage of dye from vessels on the surface of the disc. The retinal vessels may show staining in the peripapillary region. But the most important point to note is the absence of leakage from the macular vasculature, which helps it to be differentiated from papilledema resulting from increased intracranial pressure.

\subsection{Visual Evoked Potentials}

VEP is useful in the setting of multiple sclerosis where there is a latency of the P100 wave and a decrease in amplitude. It may be abnormal in neuroretinitis.

\subsection{Electroretinogram}

ERG assesses the functional integrity of the retinal layers and hence normal in disorders involving ganglion cells and optic nerve as in optic neuritis and neuroretinitis.

\section{CLINICAL COURSE}

Neuroretinitis is usually a self-limited disorder with a good visual prognosis. Typically over 6 to 8 weeks, the optic disc swelling resolves and the appearance of the disc becomes normal or mildly pale. The macular exudates appear late and progress over about 7 to 10 days, then remain stable for several weeks before gradual resolution occurs over 6 to 12 months. Most patients ultimately recover good visual acuity, although some complain of persistent metamorphopsia or nonspecific blurred vision from mild disruption of the macular architecture. In a series of Dreyer et al., visual acuity was 20/20 or better in $66 \%$ of cases, $20 / 25$ to $20 / 40$ in $31 \%$ [7]. Macular star resolves with time leaving behind RPE atrophic patches.

Most patients do not experience a subsequent attack in the same eye, and only a few patients develop a similar attack in the fellow eye. Neuroretinitis is a disease of children and young adults and the occurrence of a similar picture in an older individual should arouse suspicion for a different diagnosis like ischemic optic neuropathy [6].

\section{TREATMENT: (TABLE 4)}

Treatment of neuroretinitis depends on whether there is

Table 4. Guidelines for treatment of neuroretinitis.

\begin{tabular}{|c|}
\hline Idiopathic neuroretinitis \\
\hline High dose oral corticosteroids \\
\hline Infectious neuroretinitis \\
\hline $\begin{array}{l}\text { Cat scratch fever: oral doxycycline }(<8 \text { years) or erythro } \\
\text { may be indicated in preventing recurrences in HIV-posit }\end{array}$ \\
\hline Lyme disease: 2 - 4 week course of i.v. ceftriaxone \\
\hline Syphilis: aqueous crystalline penicillin G: 18 - 24 millio \\
\hline Toxoplasmosis: it is self-limiting disease in non-AIDS p \\
\hline Varicella zoster: immediate institution of iv acyclovir the \\
\hline Recurrent neuroretinitis \\
\hline High dose intravenous or oral steroids \\
\hline Taper oral steroids to $10 \mathrm{mg}$ alternate days \\
\hline Consider azathioprine for long term immunosupression \\
\hline
\end{tabular}


an underlying infectious or inflammatory condition that requires therapy. No treatment is required in the idiopathic group as the disease is self-limiting. Cat-scratch disease is usually described as a benign, self-limited illness [19]. Patients with neuroretinitis associated with cat scratch disease have been treated with prednisolone, dexamethasone, clindamycin, ciprofloxacin, trimethoprim-sulfa, or tetracycline and all had improved vision $[20,21]$. Doxycycline and rifampin appear to shorten the course of disease and hasten visual recovery. Long-term prognosis is good, but some individuals may acquire a mild postinfectious optic neuropathy.

Patients with neuroretinitis and secondary or late syphilis should be treated with intravenous penicillin, and patients with Lyme disease should also be treated with an appropriate antibiotic such as ceftriaxone, amoxycillin, or tetracycline. Though systemic steroids have been tried, there is no definite evidence that such treatment alters either the speed of recovery or the ultimate outcome. The prognosis in most cases of idiopathic neuroretinitis is excellent as it is self limiting [22].

\section{CONCLUSION}

Thus in most cases, neuroretinitis represents a self-limiting, benign, systemic inflammatory process with rarely a specific etiology being identified. The extent of diagnostic examination should be predicted based on the presence or absence of associated constitutional symptoms. Vision should be expected to recover within weeks to months. Nevertheless, the ophthalmologist should use caution in predicting ultimate visual prognosis.

\section{REFERENCES}

[1] Leber T. (1916) Die pseudonephritischen netzhauterkrankungen, die retinitis stellata: Die purtschersche netzhautaffektion nack schwerer schadelverletzung. In: Graefe, A.C. and Saemische, T., Eds., Graefe-Saemisch Handbuch der Augerheilkunde, 2nd Edition, Engelmann, Leipzig, 1319.

[2] Gass, J.D.M. (1977) Diseases of the optic nerve that may simulatemacular disease. Transactions American Academy of Ophthalmology and Otolaryngology, 83, 766-769.

[3] Maitland, C.G. and Miller, N.R. (1984) Neuroretinitis. Archives of Ophthalmology, 102, 1146-1150. http://dx.doi.org/10.1001/archopht.1984.01040030924014

[4] Walsh, F.B. and Hoyt, W.F. (1982) Neuroretinitis. In: Clinical Neuro-Ophthalmology, 3rd Edition, Williams \& Wilkins Co., Baltimore, 234-235.

[5] Glaser, J.S. (1978) Neuro-ophthalmology. Harper \& Row Publishers Inc., Hagers-Town, 85.

[6] Purvin, V., Sundaram, S. and Kawasaki, A. (2011) Neuroretinitis: Review of literature and new observations. Journal of Neuro-Ophthalmology, 31, 58-68. http://dx.doi.org/10.1097/WNO.0b013e31820cf78a
[7] Fish, R.H., Hoskins, J.C. and Kline, L.B. (1993) Toxoplasmosis neuroretinitis. Ophthalmology, 100, 1177-1182.

[8] Purvin, V., Ranson, N. and Kawasaki, A. (2003) Idiopathic recurrent neuroretinitis-Effects of long-term immunosuppression. Archives of Ophthalmology, 121, 6567. http://dx.doi.org/10.1001/archopht.121.1.65

[9] Folk, J.C., Weingeist, T.A., Corbett, J.J., Lobes, L.A. and Watzke, R.C. (1983) Syphilitic neuroretinitis. American Journal of Ophthalmology, 95, 480-486.

[10] Foster, R.E., Lowder, C.Y., Meisler, D.M., Kosmorsky, G.S. and Baetz-Greenwalt, B. (1990) Mumps neuroretinitis in an adolescent. American Journal of Ophthalmology, 110, 91-93.

[11] Fusco, R., Magli, A. and Guacci, P. (1986) Stellate maculopathy due to salmonella typhi: A case report. Ophthalmologica, 192, 154-158.

http://dx.doi.org/10.1159/000309629

[12] Duke-Elder, S. and Dobree, J.H. (1967) Diseases of the retina. In: Duke-Elder, Ed., System of Ophthalmology, Vol. 10, Henry Kimpton, London, 126-127.

[13] Bird, A.C., Smith, J.L. and Curtin, V.T. (1983) Nematode optic neuritis. American Journal of Ophthalmology, 95, 480-486.

[14] Forooghian, F., Lam, W.C., Hopkins, J. and Dhanda, D. (2005) Bilateral Neuroretinitis with peripapillary serous retinal detachments in a patient with HIV and HBV. Archives of Ophthalmology, 123, 1447-1449. http://dx.doi.org/10.1001/archopht.123.10.1447

[15] Parmley, V.C., Schiffman, J.S., Maitland, C.G., Miller, N.R., Dreyer, R.F. and Hoyt, W.F. (1987) Does neuroretinitis rule out multiple sclerosis? Archives of Neurology, 44, 1045-1048.

http://dx.doi.org/10.1001/archneur.1987.00520220047015

[16] Williams, K.E. and Johnson, L.N. (2004) Neuroretinitis in patients with multiple sclerosis. Ophthalmology, 111, 335-340.

http://dx.doi.org/10.1016/S0161-6420(03)00663-8

[17] Leavitt, J.A., Pruthi, S. and Morgenstern, B.Z. (1997) Hypertensive retinopathy mimicking neuroretinitis in a twelve-year-old girl. Survey of Ophthalmology, 41, 477480. http://dx.doi.org/10.1016/S0039-6257(97)00016-7

[18] Balcer, L.J. (2006) Optic neuritis. New England Journal of Medicine, 354, 1273-1280. http://dx.doi.org/10.1056/NEJMcp053247

[19] Carithers, H.A. (1985) Cat-scratch disease. An overview based on a study of 1200 patients. American Journal of Diseases of Children, 139, 1124.

[20] Golnik, K.C., Marotto, M.E., Fanous, M.M. and Heitter, D. (1994) Ophthalmic manifestations of rochalimaea species. American Journal of Ophthalmology, 118, 145-151.

[21] Chrousos, G.A., Drack, A.V., Young, M., Kattah, J. and Sirdofsky, M. (1990) Neuroretinitis in cat-scratch disease. Journal of Clinical Neuro-Ophthalmology, 10, 92-94.

[22] Dreyer, R.F., Hopen, G., Gass, J.D.M. and Smith, J.L. (1984) Leber's idiopathic stellate neuroretinits. Archives of Ophthalmology, 102, 1140-1145. http://dx.doi.org/10.1001/archopht.1984.01040030918013 